\title{
Are Simple Real Pole Solutions Physical?
}

\author{
M. Berg*† \\ Center for Relativity \\ University of Texas at Austin, USA \\ M. Bradley ${ }^{\ddagger}$ \\ Department of Plasma Physics \\ Umeå University, Umeå, Sweden
}

November 20, 2018

PACS Ref: 04.20.Jb, 04.30.Nk, 04.40.Nr

\begin{abstract}
We consider exact solutions generated by the inverse scattering technique, also known as the soliton transformation. In particular, we study the class of simple real pole solutions. For quite some time, those solutions have been considered interesting as models of cosmological shock waves. A coordinate singularity on the wave fronts was removed by a transformation which induces a null fluid with negative energy density on the wave front. This null fluid is usually seen as another coordinate artifact, since there seems to be a general belief that that this kind of solution can be seen as the real pole limit of the smooth solution generated with a pair of complex conjugate poles in the transformation. We perform this limit explicitly, and find that the belief is unfounded: two coalescing complex conjugate poles cannot yield a solution with one real pole. Instead, the two complex conjugate poles go to a different limit, what we call a "pole on a pole". The limiting procedure is not unique; it is sensitive to how quickly some parameters approach zero. We also show that there exists no improved coordinate transformation which would remove the negative energy density. We conclude that negative energy is an intrinsic part of this class of solutions.
\end{abstract}

$\mathrm{gr}-\mathrm{qc} / 0002042$

\footnotetext{
*This work was partially performed while visiting the Dept. of Plasma Physics at Umeå

${ }^{\dagger}$ Electronic mail: mberg@einstein.ph.utexas.edu

‡Electronic mail: michael.bradley@physics.umu.se
} University. 


\section{Introduction}

\section{$1.1 \quad$ Background}

We consider exact solutions generated by the inverse scattering technique, also known as the soliton transformation, in the so-called cosmological case (see section 2 below). We do not consider the stationary axisymmetric case since we are interested in solutions of wave-like character and cosmological application. A comprehensive review of this subject and applications related to that of this paper has been given by Verdaguer [1]. In the review one also finds references to earlier work in the field by Ernst, Chandrasekhar and others in the 1970s and 1980s. A general discussion of waves modelled by exact solutions is given in the book by Griffiths [2].

The inverse scattering technique was first applied to general relativity in 1978 by Belinskii and Zakharov [3]. Their algorithm generates exact vacuum solutions to Einstein's equations from a known "seed solution", also called the "background". This background is multiplied by the so-called scattering matrix to yield the new metric, called the perturbation. Note that the use of the word perturbation does not imply an approximation, the new metric is still an exact solution. Any poles in the scattering matrix give rise to solitonic solutions, and these have earned the inverse scattering technique its other name "soliton transformation". The transformation is closely related to the Bäcklund transformations, and the exact relation has been clarified by Cosgrove 4 , 5.

\subsection{Simple real pole solutions}

The inverse scattering technique can recreate well-known solutions. One interesting example is the Kerr-NUT metric generated from flat space [6] with two real poles in the scattering matrix. Homogenous cosmologies of Bianchi types I through VII can be generated with the inverse scattering technique. New solutions have also been produced, often with gravitational solitons. One class of such solutions that has been studied extensively [1, 3, 7, 8, 9] is the "one real pole" perturbation on a Bianchi I or II background (for the purposes of this article, we choose the Kasner vacuum metric as a background). One real pole solutions may have merits as cosmological models; to see this we briefly survey the physical behavior of the solutions.

The relevant region for cosmology is $t>0$ (see fig. 1). A solitary shock wave emerges from the initial singularity at $z=w_{0}, t=0$. Behind the wavefront, the shock wave leaves way for the background (Kasner) metric.

Thus this soliton-like disturbance sweeps away some initial disorder which surrounds the singularity. This has earned the single real pole solution generated from a Bianchi I or II metric its nickname "the cosmic broom". (From the Italian la scopa cosmica.) Simple real pole solutions generated from other seed metrics or with more poles display similar behavior. The cosmic broom represents an interesting exact-solution model of gravitational waves of cosmological origin. A background of such waves could in principle be detected, and the cosmic broom 


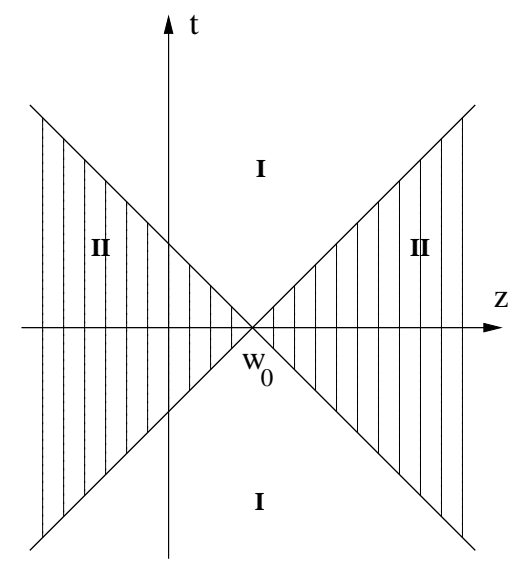

Figure 1: The light cone structure. Pole at $w_{0}$.

would provide an intriguing classical alternative to waves generated from the initial conditions of the universe in quantum cosmology.

Furthermore, it turns out that the perturbation is undefined in region I, so we match the perturbation in region II across the light cone to the background (Kasner) metric in region I. While other choices for the matching are possible, we match to the background for two main reasons. One reason is that the perturbed metric in region II itself goes into the unperturbed one when approaching the light cone. The other reason is that we wish to maintain the traveling wave interpretation, which is most meaningful when the metric is asymptotically equal to the background on both sides of the disturbance.

In general, one real pole is not sufficient to recover the background at spatial infinity. Instead, this can be achieved with two or more distinct real poles [1]. Since each real pole defines a light cone, one then has to perform a number of matchings. Some of these matchings will involve matching the background within the inner light-cone to one-pole solutions. Consequently, a first step is to match the background to a one-pole solution, even though one is ultimately interested in $n$-pole solutions or a stochastic background of waves. Therefore, the problem described in this paper can be seen as part of the more complicated matching procedure for several distinct real poles.

A good motivation to use a Kasner-type background is that it has been shown that any "generic" singularity is followed by a succession of Kasner eras [10]. Moreover, a Bianchi I metric is approximately unaffected close to the singularity by the presence of matter 11. (Recently, doubt has been cast on this somewhat folkloristic claim [12].) 


\subsection{Problems}

The cosmic broom suffers from metric coefficient infinities on the light cone in canonical (Belinskii-Zakharov) coordinates. Recently it was shown that these coordinate infinities can be removed by a certain (singular) coordinate transformation [8, 9]. However, a new but potentially less serious problem was created by this transformation. Although the metric is now continuous, the Ricci tensor goes distributional-valued, i.e. acquires $\delta$-functions, at the light cone. This kind of $\delta$-function can normally be interpreted as an impulsive wave, e.g. a null fluid. (see the book by Griffiths 22 on the subject). Unfortunately, the null fluid in this case would have negative energy density [8]. Let us exhibit this explicitly for the left hand side of the light cone $\left(z<w_{0}\right)$, a similar discussion holds for the right hand side. From Einstein's equation with the aforementioned Ricci tensor one finds

$$
T_{i j}=-\frac{1}{8 \pi t g_{t t}} \delta\left(t+z-w_{0}\right) v_{i} v_{j}
$$

where $v^{a}=1 / \sqrt{2 g_{t t}}(\partial / \partial t-\partial / \partial z)$ is along the light cone. An observer at constant $z<w_{0}$ with 4 -velocity $\xi^{a}=\left(1 / \sqrt{g_{t t}}\right) \partial / \partial t$ would measure the following energy density when the wave front passes:

$$
\rho=T_{i j} \xi^{i} \xi^{j}=-\frac{1}{16 \pi t g_{t t}} \delta\left(t+z-w_{0}\right)<0
$$

which is manifestly negative and thus violates the weak energy condition. (Our signature is $(+---)$ as is usual for inverse scattering applications, so $g_{t t}>0$. Also we are at $t>0$.) Therefore, one would naively think that the present "null fluid" is merely an artifact of the singular transformation and that the "actual" one real pole solution has no null fluid. (See also work by Gleiser and Díaz 13, 14, 15 on the problem of matching solutions in different regions and for methods of removing the singularities on the light-cone and obtaining smooth extensions.)

There are at least two ways to find a more physical form of the one real pole solutions. The most obvious is to "sew together" the coordinate patches in a different way, i.e. to find a better transformation which is non-singular but still removes the coordinate infinites which the metric has in canonical coordinates. The coordinate transformation is discussed in section 4 .

As an alternative, there seems to be a general belief ([1], sec. 3.7.1) that the one real pole solution be sought as the real limit of two complex conjugate poles $w_{0} \pm i \epsilon$. The complex conjugate pole solution is known to be smooth everywhere. Indeed, this limiting procedure has often been implicitly invoked to support the validity of simple real pole solutions such as the cosmic broom. (The reason one does not simply use the complex pole solution directly is that it contains disturbances which travel at $v>c$, so it in itself does not admit a traveling wave interpretation similar to that of the real pole solutions, where the shock wave is strictly located to the light cone and thus travels at $v=c$.) The limiting procedure is the subject of section 3 .

Our conclusions are that 
- The limiting procedure actually yields a different solution, so it cannot be used to explain away the null fluid, and

- There is no improved transformation, so this avenue out is also closed.

\section{Setup}

We use the conventions and notation of Belinskii and Francaviglia 7 . The inverse scattering technique is applicable for vacuum metrics of the form

$$
\mathrm{d} s^{2}=f(z, t)\left(\mathrm{d} t^{2}-\mathrm{d} z^{2}\right)-g_{a b}(z, t) \mathrm{d} x^{a} \mathrm{~d} x^{b}, \quad a, b=1,2,
$$

which describes a spacetime admitting an Abelian $G_{2}$ group of isometries [3]. Examples of such metrics are given in the introduction. This form of the metric is in the "cosmological case". The other (stationary axisymmetric) case, which we do not consider, is related to this one by a complex transformation, but the stationary axisymmetric solutions are quite different in character. In the following $g$ is the $2 \times 2$ matrix representation of $g_{a b}$.

Belinskii and Francaviglia [7] found an explicit expression for $g$ and $f$ of the complex conjugate pole solution. Note that we have replaced $\alpha=t$ in their expression. Also note that the superscripts "(2)" and "(0)" refer to "two poles" and "no poles" (background), respectively. A bar represents complex conjugation.

$$
\begin{aligned}
g_{a b}^{(2)} & =g_{a b}^{(0)}+\frac{1}{\mathcal{D}}(\bar{\mu}-\mu)\left(t^{2}-|\mu|^{2}\right)\left[\frac{1}{\mu}\left(t^{2}-\mu^{2}\right) Q_{22} L_{a} L_{b}\right. \\
& \left.-\frac{1}{\bar{\mu}}\left(t^{2}-\mu^{2}\right) Q_{11} \bar{L}_{a} \bar{L}_{b}-(\bar{\mu}-\mu) Q_{22} Q_{11} g_{a b}^{(0)}\right] \\
f^{(2)} & =c_{2} \frac{(\mu \bar{\mu})^{3} \mathcal{D}}{\left(t^{2}-\mu \bar{\mu}\right)^{2}\left(t^{2}-\mu^{2}\right)\left(t^{2}-\bar{\mu}^{2}\right)(\mu-\bar{\mu})^{2} t^{2}} f^{(0)}
\end{aligned}
$$

where the vector $L_{a}$ and the scalars $Q_{k l}$ are

$$
\begin{aligned}
L_{1} & =m_{1} g_{11}^{(0)}, \quad L_{2}=m_{2} g_{22}^{(0)} \\
Q_{11} & =m_{1}^{2} g_{11}^{(0)}+m_{2}^{2} g_{22}^{(0)}, \\
Q_{12} & =m_{1} \bar{m}_{1} g_{11}^{(0)}+m_{2} \bar{m}_{2} g_{22}^{(0)}=Q_{21} \\
Q_{22} & =\bar{m}_{1}^{2} g_{11}^{(0)}+\bar{m}_{2}^{2} g_{22}^{(0)}=\bar{Q}_{11}
\end{aligned}
$$

the $m_{1}$ and $m_{2}$ are determined by the seed metric ( $s$ is the Kasner parameter):

$$
m_{a}=\left(m_{01}(2 w \mu)^{-s}, \quad m_{02}(2 w \mu)^{s-1}\right)
$$

with $m_{0 b}$ arbitrary complex constants, and $\mathcal{D}$ is found from

$$
\begin{aligned}
& \Delta=Q_{11} Q_{22}-Q_{12}^{2}=t^{2}\left(m_{1} \bar{m}_{2}-\bar{m}_{1} m_{2}\right)^{2} \\
& \mathcal{D}=t^{2} Q_{11} Q_{22}(\mu-\bar{\mu})^{2}+\Delta\left(t^{2}-\mu^{2}\right)\left(t^{2}-\bar{\mu}^{2}\right) .
\end{aligned}
$$


The pole "trajectory" $\mu(z, t)$, called trajectory because it depends on $z$ and $t$, is

$$
\mu(z, t)=w-z \pm \sqrt{(w-z)^{2}-t^{2}}
$$

where $w=w_{0}+i \epsilon$. (In the original inverse scattering method, before it was applied to general relativity, the poles were constants.) It is of interest to note that the pole trajectory is the result of setting the coefficients of any second order poles to zero, so the method is by construction only treating simple poles.

For the Kasner metric, we note finally

$$
\begin{aligned}
& g_{11}^{(0)}=t^{2 s}, \quad g_{22}^{(0)}=t^{2-2 s} \\
& f^{(0)}=t^{2 s^{2}-2 s}
\end{aligned}
$$

Now that the complete metric is given, it is of interest to count the free parameters: the complex pole $w$ (eq. 6), the two complex parameters $m_{0 a}$ (eq. (4) and the real scaling factor $c_{2}$ (eq. 3), making for seven real parameters.

We will also need the expression for the one real pole solution [8] (recall the superscript "(1)" indicates one pole):

$$
\begin{aligned}
& g_{a b}^{(1)}=\frac{|\tilde{\mu}|}{t}\left(g_{a b}^{(0)}+\frac{t^{2}-\tilde{\mu}^{2}}{\tilde{\mu}^{2} Q} \tilde{L}_{a} \tilde{L}_{b}\right) \\
& f^{(1)}=c_{1} \frac{\tilde{\mu}^{2} Q}{\left(t^{2}-\tilde{\mu}^{2}\right) \sqrt{t}} f^{(0)}
\end{aligned}
$$

with the auxiliary quantities

$$
\begin{aligned}
\tilde{m}_{a} & =\left(\tilde{m}_{01}\left|2 w_{0} \tilde{\mu}\right|^{-s}, \quad \tilde{m}_{02}\left|2 w_{0} \tilde{\mu}\right|^{s-1}\right) \\
\tilde{L}_{a} & =\left(\tilde{m}_{1} g_{11}^{(0)}, \quad \tilde{m}_{2} g_{22}^{(0)}\right) \\
Q & =\tilde{m}_{1}^{2} g_{11}^{(0)}+\tilde{m}_{2}^{2} g_{22}^{(0)} \\
\tilde{\mu} & =w_{0}-z \pm \sqrt{\left(w_{0}-z\right)^{2}-t^{2}}
\end{aligned}
$$

We will see that these are real parts of the corresponding variables for $g^{(2)}$ above, e.g. $L_{a} \rightarrow \tilde{L}_{a}$ as $\epsilon \rightarrow 0$. Again we count the parameters and find only $\tilde{m}_{0 a}, c_{1}$ and $w_{0}$, four real parameters.

\section{The real limit}

\subsection{General conclusions}

The procedure for taking the limit is not unique. For instance, let us take the expression for the $2 \times 2$ scattering matrix which generates the transformation, given by Belinskii and Zakharov [3] as

$$
\chi(\lambda, z, t)=1+\frac{R_{1}}{\lambda-\mu}+\frac{R_{2}}{\lambda-\bar{\mu}}
$$


where $R_{1}$ and $R_{2}$ are $2 \times 2$ matrices and $\lambda$ is the spectral parameter. Now, if $\mu \rightarrow \bar{\mu}$ as $\epsilon \rightarrow 0$, we would obtain

$$
\chi(\lambda, z, t)=1+\frac{R_{1}+R_{2}}{\lambda-\mu} \quad,
$$

indistinguishable from the expression for one real pole. This seems to verify that the limiting procedure will, indeed, produce a simple real pole. However, one should note that the original expression (8) was intended for distinct poles [3].

Instead of taking the limit in (8) we consider the behavior of the actual metric tensor as the complex conjugate poles $w_{0} \pm i \epsilon$ approach the real pole $w_{0}$ by sending $\epsilon \rightarrow 0$ (see fig. 2).

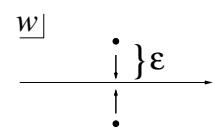

Figure 2: Taking the limit $\epsilon \rightarrow 0$ in $w=w_{0} \pm i \epsilon$.

It is useful to discuss regions I (inside the light cone $\left.\left(w_{0}-z\right)^{2}<t^{2}\right)$ and II (outside the light cone $\left.\left(w_{0}-z\right)^{2}>t^{2}\right)$ separately. Refer to fig. 1 for the following discussion.

i) Region $\mathbf{I}$

From equation (6), region I will never have a real pole trajectory, but instead $g$ approaches the background. Indeed, from that equation one finds

$$
|\mu|^{2} \rightarrow t^{2} \quad \text { when } \quad \epsilon \rightarrow 0 \quad \text { (region I) , }
$$

which implies that $g$ approaches the background. This in itself would suggest that we match the solution to the background in region I. On the other hand,

$$
g_{t t}=-g_{z z}=f(z, t ; \epsilon) \rightarrow \infty \quad \text { as } \quad 1 / \epsilon^{2} \quad(\text { region } \mathrm{I}) \quad,
$$

but as long as $\epsilon$ is nonzero, one can still rescale $f$ using the scaling constant $c_{2}$ in equation (3) to allow for matching.

ii) Region II

We now expand the pole trajectory $\mu$ (eq. 6) in region II and find

$$
\mu(z, t) \rightarrow \tilde{\mu}(z, t)+i \kappa(z, t) \epsilon \quad(\text { region II }) \quad,
$$

where $\kappa(z, t)$ does not approach zero as $\epsilon \rightarrow 0$. Thus the complex conjugate pole trajectory does approach the corresponding trajectory for the real pole solution. 
Now for the metric itself in region II. Special attention must be paid to the parameters; as was noted earlier, simple real pole solutions have only four real parameters while the complex pole solution has seven. We know the pole $w$ becomes real. The two complex parameters $m_{0 a}$ must become real in the limit, which accounts for the difference. An obvious ansatz for $m_{0 a}$ is

$$
m_{0 a}=\tilde{m}_{0 a}+i \epsilon^{p}
$$

i.e. to let the complex parameters approach the corresponding real parameters as some power $p$ of $\epsilon$. With this ansatz, it can be seen from equation (5) that

$$
\mathcal{D} \rightarrow 0 \quad \text { as } \quad a \epsilon^{2}+b \epsilon^{2 p} \quad(\text { region II) } \quad,
$$

where $a$ and $b$ do not approach zero.

We find two different cases: for $p<1$, the second term in (2) vanishes, since $\mathcal{D}$ approaches zero slowly - we are left with only the background in region II (as well as region I). On the other hand, for $p>1$ we do obtain a new solution in region II.

These results differ somewhat from earlier assertions [7], which state that the parameters must be proportional to each other, otherwise a finite perturbation cannot be found in the limit. This is again related to the issue of the number of free parameters, which is reduced when the parameters are taken to be proportional. Complex conjugate parameters can never be proportional, so if one takes this claim literally the limiting procedure can only be applied to the case of two real poles. However, in this argument the behavior of the parameters $m_{0 a}$ was not considered, and our scheme relies on the fact that these parameters approach reality with the poles, so it is free of this restriction. In [16] it is shown that a double pole can be obtained from two coalescing poles if once again the parameters are proportional, but the paper does not study the question whether single poles can be formed or not. Neither [7] nor [16] treated the possibility of taking the real limit of complex constants.

Now we turn to discuss the limit in detail. The general non-diagonal case becomes rather cumbersome, so we perform the limit numerically. As an instructive example, we begin by studying the diagonal case analytically.

\subsection{The diagonal case}

A diagonal perturbation is obtained by taking, for example, $m_{02}=0$. For a pair of distinct complex conjugate poles, we find

$$
\begin{aligned}
g_{11} & =\frac{t^{2}}{\mu \bar{\mu}} g_{11}^{(0)}, \quad g_{22}=\frac{\mu \bar{\mu}}{t^{2}} g_{22}^{(0)}, \\
f & =c_{2} \frac{|\mu|^{6}\left(g_{11}^{(0)}\right)^{2}}{|2 w \mu|^{4 s}\left(t^{2}-|\mu|^{2}\right)^{2}\left(t^{2}-\mu^{2}\right)\left(t^{2}-\bar{\mu}^{2}\right)} f^{(0)}
\end{aligned}
$$


which is known as the Einstein-Rosen metric [1]. At this point, one notes that the exact same expression applies to the case of two real poles if $\mu_{1}$ and $\mu_{2}$ are substituted for $\mu$ and $\bar{\mu}$, respectively. If we take the diagonal perturbation for one real pole, on the other hand, we find from equation $(\bar{\nabla})$ :

$$
\begin{aligned}
g_{11} & =\frac{t}{|\mu|} g_{11}^{(0)}, \quad g_{22}=\frac{|\mu|}{t} g_{22}^{(0)}, \\
f & =c_{1} \frac{\mu^{2} g_{11}^{(0)}}{\left|2 w_{0} \mu\right|^{2 s}\left(t^{2}-\mu^{2}\right) \sqrt{t}} f^{(0)}
\end{aligned}
$$

By inspection, we cannot obtain this metric from (9) by $\epsilon \rightarrow 0$. On the other hand, the two real pole metric has exactly the form (9). Therefore, if the limit is taken at this stage, one does not obtain a simple real pole but two confluent real poles. This conclusion agrees with an older study 17, which was limited to the present special case of a diagonal perturbation. The solution is, in general, not the two real pole solution itself since there is again ambiguity in the way the parameters coalesce. In addition, the expression for two real poles was intended for distinct poles, but this solution clearly exists as a limit. This is what we mean by "a pole on a pole".

\subsection{The non-diagonal case}

We perform the limit numerically in the non-diagonal case. Details are available elsewhere 18 , but the salient points are summarized here. We find that conclusions from the diagonal case carry through to the non-diagonal case. The metric for complex conjugate poles approaches the background everywhere for $p<1$, but approaches a finite perturbation for $p>1$, just as asserted on analytical grounds earlier by studying $\mathcal{D}$. An example of this is given in fig. 3 for $p=2$. The two complex pole solution clearly does not approach the one real pole solution.

It appears that the two complex pole solution approaches the two real pole solution in the limit instead. However, the particular parameter dependence (given in the caption) has been chosen such that this would happen; in general the limit is different, although it is true that the limit procedure shown in fig. 3 may be thought of as the most natural limit for this comparison. All this is in agreement with the conclusion in the diagonal case.

It is easy to see numerically that the metric coefficient $f$ does approach infinity as $\epsilon^{-2}$. Belinskii and Francaviglia point out that if the scaling constant $c_{2}$ is replaced with (essentially) $\epsilon^{2}$, we can match $f$ to the background on the light cone[7. This would seem to allow for a finite $f$ in the limit, since $\lim _{\epsilon \rightarrow 0} \epsilon^{2} / \epsilon^{2}=1$. Unfortunately, when $\epsilon=0$, this would also mean $c_{2}=0$. Since there is only one scaling constant, the metric would then become degenerate in region II, where $f$ does not approach infinity.

We conclude that the limit of two complex conjugate poles does produce a solution, but it is not the one real pole solution. Furthermore, this "pole on a pole" solution also suffers from the deficiency of being infinite inside the light 

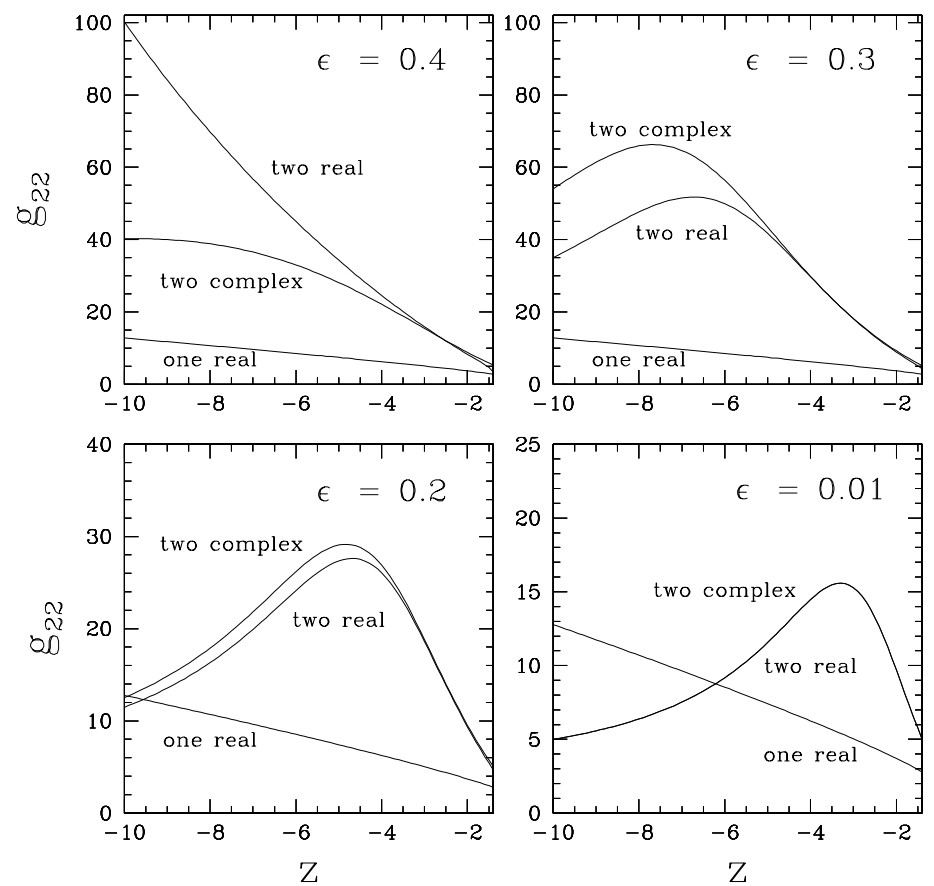

Figure 3: Taking $\epsilon \rightarrow 0$ for $w=1+i \epsilon, m_{0 \mu}=\left(1+i \epsilon^{2}, 2+i \epsilon^{2}\right), s=0.6, t=2$. For the two real pole solution we take $w_{ \pm}=1 \pm \epsilon, m_{0 \mu}^{( \pm)}=\left(1 \pm \epsilon^{2}, 2 \pm \epsilon^{2}\right)$ which is a natural choice compared to the former.

cone. Thus, the problem of finding a coordinate transformation which removes the infinity in $f$ remains for the one real pole solution.

\section{Nonexistence of improved transformations}

Instead of the limit procedure above, one could attempt to improve the coordinate transformation for a simple real pole which has been used by several authors 8, 9]. A nonsingular transformation, which still does the job of rendering $f$ finite, would eliminate the null fluid.

We here briefly summarize the results from Curir and one of the authors [8]. In terms of null coordinates

$$
u=t+z-w_{0}, \quad v=t-z+w_{0},
$$

the metric can be written as

$$
\mathrm{d} s^{2}=f \mathrm{~d} u \mathrm{~d} v-g_{a b} \mathrm{~d} x^{a} \mathrm{~d} x^{b}, \quad a, b=1,2 .
$$


When approaching the light cone $u=0$ from the perturbed region II, $f$ goes to infinity as $1 / \sqrt{-u}$ ( $v=0$ can be treated in a similar way). By changing coordinate from $u$ to

$$
u^{\prime}=\mp \frac{c_{1} Q_{0}}{\sqrt{2}} \sqrt{-u}
$$

where $Q_{0}$ is the value of $Q$ on the light cone, the metric changes to

$$
\mathrm{d} s^{2}=f^{\prime} \mathrm{d} u^{\prime} \mathrm{d} v-g_{a b} \mathrm{~d} x^{a} \mathrm{~d} x^{b},
$$

where $f^{\prime}$ is finite on the light cone and matches with $f^{(0)}$ from the unperturbed region I: $f^{\prime}\left(u^{\prime}=0\right)=f^{(0)}(u=0)$. (By defining $u^{\prime}=u$ in the unperturbed region $\mathrm{I}$, one sees that the light cone still is given by $u^{\prime}=0$ and that $u^{\prime}$ is continuous across the light cone.) All metric components are now continuous, but their first derivatives suffer step function jumps across the light cone, giving rise to delta-functions both in the Weyl tensor and the Ricci tensor.

So far points with the same value of $u^{\prime}$ and $v$ have been identified along the light cone. Could one make the discontinuities disappear by identifying them in some other way? We have the following result:

Proposition 1 There is no (topology-preserving) coordinate transformation of the one real pole solution (eq. (7) which both makes $f$ everywhere finite and removes the $\delta$-functions in the Ricci tensor.

We proceed to show this. Clearly the light cone is given by $u^{\prime}=0$, so the remaining freedom is to identify different $v$ with each other. But since the 2-dimensional matrix $g_{a b}$ is continuous across the light cone one finds

$$
g_{a b I}\left(u^{\prime}=0, v_{I}\right)=g_{a b I I}\left(u^{\prime}=0, v_{I I}\right),
$$

implying that $v_{I}=v_{I I}$. New coordinates $\tilde{u}$ and $\tilde{v}$ (we choose to perform a transformation in region II only, since this is general enough) must then satisfy

$$
\tilde{u}(0, v)=u^{\prime}=0, \quad \tilde{v}(0, v)=v,
$$

on the light cone. Allowed transformations can then be written as

$$
u^{\prime}=\tilde{u}+F(\tilde{u}, \tilde{v}), \quad v=\tilde{v}+G(\tilde{u}, \tilde{v}),
$$

where $F$ and $G$ are zero on the light cone but otherwise arbitrary functions. The requirement that the metric remains continuous then gives that $F$ and $G$ must approach zero faster than $\tilde{u}$. On the other hand, to avoid the discontinuities giving rise to the delta-function in the Ricci-tensor, $G$ must approach zero as $\tilde{u}$ or $F$ must go as $\sqrt{-\tilde{u}}$. Hence the we cannot avoid a distributional-valued Ricci tensor by this transformation.

We do not consider transformations which induce topology change, such as rotating the light cone before identifying points. Such transformations would create completely different spacetimes than the ones we consider here.

Our conclusion is that the null fluid is a property of the one real pole solution which cannot be removed. 


\section{Conclusion}

The original idea of eliminating the null fluid in the one real pole solution through a limit of two complex conjugate poles $w=w_{0} \pm i \epsilon$ is actually impossible to realize. We reach a different solution, "a pole on a pole" instead of a simple

real pole solution. Also, we found that care must be exercised in the taking of the limit since the parameters must turn real. This is evidenced by the fact that in one case (what we call $p<1$ ) the inverse scattering transformation reduces to the identity transformation.

Additionally, we show that there is no transformation which accomplishes the goal of removing the metric infinities while keeping the null fluid away. Therefore, barring new topologies, the simple real pole solution really does contain a null fluid with negative energy density. We find this property unphysical in a completely classical solution, one would therefore reject these solutions. However, a more liberal interpretation would be that negative energy on the wave fronts is a characteristic of cosmological waves, and that this is a sign that quantum effects in the early universe are required to produce cosmological shock waves if such waves are produced at all.

Since the metric in region I approaches the background under the limiting procedure, we found it natural to match region II to the background. It makes the wave interpretation more meaningful since the metric will be asymptotically Kasner on both sides of the wave front. It is also common to do so[1]. However, it is of course possible to match the solution in region II to some metric other than the background. See e.g. [2, 19] for treatments where solutions generated by the inverse scattering technique from Bianchi models are matched to metrics other than the background.

Higher-order (i.e. non-simple) poles in the scattering matrix could also generate viable solutions, but that would be a different story altogether.

\section{Acknowledgements}

We wish to thank Lawrence C. Shepley for valuable structural advice, Matthew W. Choptuik for constructive criticism, and Lennart Stenflo at Umeå University for hospitality.

\section{References}

[1] Verdaguer E., Phys. Reports 229, No. 1-2 (1993)

[2] Griffiths J. B., "Colliding Plane Waves in General Relativity" (Oxford S.P., Oxford, 1991)

[3] Belinskii V. A. and Zakharov V. E., Sov. Phys. JETP 48, 6 (1978)

[4] Cosgrove C. M., J. Math. Phys. 22, 2624 (1981) 
[5] Cosgrove C. M., J. Math. Phys. 23, 615 (1982)

[6] Belinskii V. A. and Zakharov V. E., Sov. Phys. JETP 50, 1 (1979)

[7] Belinskii V. A. and Francaviglia M., Gen. Rel. Grav. 14, 213 (1982)

[8] Bradley M. and Curir A., Gen. Rel. Grav. 25, 539 (1993)

[9] Curir A., Francaviglia M. and Verdaguer E., Astrophys. J. 397, 390 (1992)

[10] Belinskii V. A., Khalatnikov I. M. and Lifshitz E. M., Adv. Phys. 19, 525 (1970)

[11] H. Stephani, "General Relativity" (Cambridge U.P., Cambridge, England, 1991)

[12] Bruni M., van Elst H. and Uggla C., gr-qc/9611005; from "Proc. of the 12th Italian Conf. of General Relativity and Gravitational Physics", (1996)

[13] Gleiser R. J., Gen. Rel. Grav. 16, 1077 (1984)

[14] Díaz M. C., Class. Quant. Grav. 2, 891 (1985)

[15] Díaz M. C. and Gleiser R. J., Gen. Rel. Grav. 20, 517 (1988)

[16] Gleiser R. J., González G. I. and Pullin J. A., Phys. Lett. A 130, 206 (1988)

[17] Verdaguer E., in "Observational and Theoretical Aspects of Relativistic Astrophysics and Cosmology", Sanz, J L and Goicoechea L J , eds. (World Scientific, Singapore, 1985)

[18] Berg M., "The Inverse Scattering Technique in General Relativity: The Real Pole Limit", Master's Thesis (Umeå University: Umeå, Sweden, 1996)

[19] Nagy G. B., Gleiser R. J. and Dagotto A. D., gr-qc/9609041 